\title{
HERRAMIENTAS DIGITALES DE EVALUACIÓN EN LÍNEA PARA MEJORAR LA APROBACIÓN EN APRENDIZAJES DINÁMICOS
}

\author{
DIGITAL ONLINE ASSESSMENT TOOLS TO IMPROVE APPROVAL IN DYNAMIC \\ LEARNING
}

Recibido: 21-septiembre-2021

Aceptado: 6 de noviembre 2021
O. A. García Cano ${ }^{1}$

A. A. Gutiérrez Reyes ${ }^{2}$

E. A. Meraz Salazar ${ }^{3}$

J.C. Reyes Torres ${ }^{4}$

\section{RESUMEN}

La educación tradicional ha ido evolucionando de manera paulatina, desde las corrientes pedagógicas hasta los métodos de enseñanza, para convertirse en lo que actualmente conocemos como educación a distancia, introduciendo nuevas tecnologías al salón de clases a través de aulas virtuales, estás traen consigo cambios en los procesos de enseñanza- aprendizaje, convirtiéndolas en modelos dinámicos, donde las evaluaciones formativas y sumativas sirven para desarrollar nuevas habilidades en los estudiantes, fortaleciendo el conocimiento de los distintos niveles educativos. Partiendo de lo anterior es como surge el estudio, del aprendizaje basado en herramientas digitales en el Instituto Tecnológico de Durango (ITD) con los estudiantes de Educación a Distancia (EAD), en la asignatura de Propiedades de los Materiales de la carrera de Ingeniería Industrial, con el objetivo de conocer el impacto que generan estas herramientas sobre el índice de aprobación. Esto llevó a realizar una comparación de años anteriores, con los presentes considerando el uso de las siguientes plataformas Mentimeter, Kahoot y Moodle, cada una con una peculiar características, como son, la primera permite crear presentaciones interactivas, agregar preguntas, cuestionarios y gráficos; la segunda crea cuestionarios de evaluación disponibles para aplicaciones móviles o versiones web; la tercera crea espacios de aprendizajes en línea, mediante cursos, que se generan con recursos y actividades, todas en su modo gratuito.

\section{PALABRAS CLAVE:}

Educación, Herramientas digitales, Evaluación, Aprendizaje.

\begin{abstract}
Traditional education has evolved gradually, from pedagogical currents to teaching methods, to become what we currently know as distance education, introducing new technologies to the classroom through virtual classrooms, these bring changes in teaching-learning processes, turning them into dynamic models, where formative and summative evaluations serve to develop new skills in students, strengthening knowledge of the different educational levels. Based on the above, the study arises, of learning based on digital tools at the Technological Institute of Durango (ITD) with Distance Education students (EAD), in the subject of Properties of Materials of the Industrial Engineering career, with the aim of knowing the impact that these tools generate on the approval rate. This led to a comparison of previous years, with those present considering the use of the following platforms Mentimeter, Kahoot and Moodle, each one with its own peculiar characteristics, such as, the first one allows creating interactive presentations, adding questions, questionnaires and graphics; the second creates evaluation questionnaires available for mobile applications or web versions; the third creates spaces for online learning, through courses, which are generated with resources and activities, all in their free mode.
\end{abstract}

\section{KEY WORDS:}

Education, Digital tolos, Assessment, Learning

\footnotetext{
${ }^{1}$ Profesor de 3/4 de tiempo. TecNM, Campus Durango, obedgarciac@itdurango.edu.mx

${ }^{2}$ Profesor de asignatura. TecNM, Campus Ciudad Valles, azucena.gutierrez@tecvalles.mx

${ }^{3}$ Profesor de tiempo completo. TecNM, Campus Lerdo, erikamerazs@ gmail.com

${ }^{4}$ Profesor de tiempo completo. TecNM, Campus Acayucan, judconreto@itsacayucan.edu.mx
} 


\section{INTRODUCCIÓN}

La educación universitaria ha sufrido cambios dentro de su estructura pedagógica y tecnológica, para desarrollar conocimientos, generando que estos sean adquiridos de manera más sencilla y a la vez práctica es por ello que requiere de docentes capaces de interactuar en distintos ámbitos, generando las destrezas adecuadas para utilizarlas en plataformas tecnológica con fines didácticos, es importante mencionar que estas herramientas conllevan evaluaciones de carácter dinámicas y significativas para los estudiantes.

Por lo que la presente investigación se centra en el uso de la tecnología como una herramienta digital que mejora los procesos de aprendizajes dinámicos considerando los diferentes tipos de evaluación para fortalecer el aprendizaje como lo son: diagnóstica, formativa y sumativa, las cuales comprueban el desarrollo del conocimiento en el estudiante. Cabe señalar que el Instituto Tecnológico de Durango cuenta con la modalidad de Educación a Distancia (EAD), mediante esta se analizaron el uso de tres plataformas digitales que pueden ser utilizadas para la evaluación de contenido en el ambiente universitario, Mentimeter, Kahoot y Moodle.

La Universidad del Pacifico (2020), en Perú indica que Mentimeter es una poderosísima herramienta web online que permite hacer preguntas, encuestas y juegos a la audiencia. En el caso de la plataforma Kahoot según el periódico El Universal en el 2019 registró 1,250 millones de participantes en más de 200 países, en la actualidad ya tiene una aplicación para mediante juegos repasar contenido interesante. Y, por último, según docs.moodle.org para el año 2021 la plataforma Moodle es utilizada por más de 294 millones de usuarios en 240 países, lo cual la convierte en la plataforma de aprendizaje más ampliamente utilizada en México y en el mundo. En la figura 1, se describe la cantidad de sitios cursos, usuarios y países a los que llegan en todo el mundo.

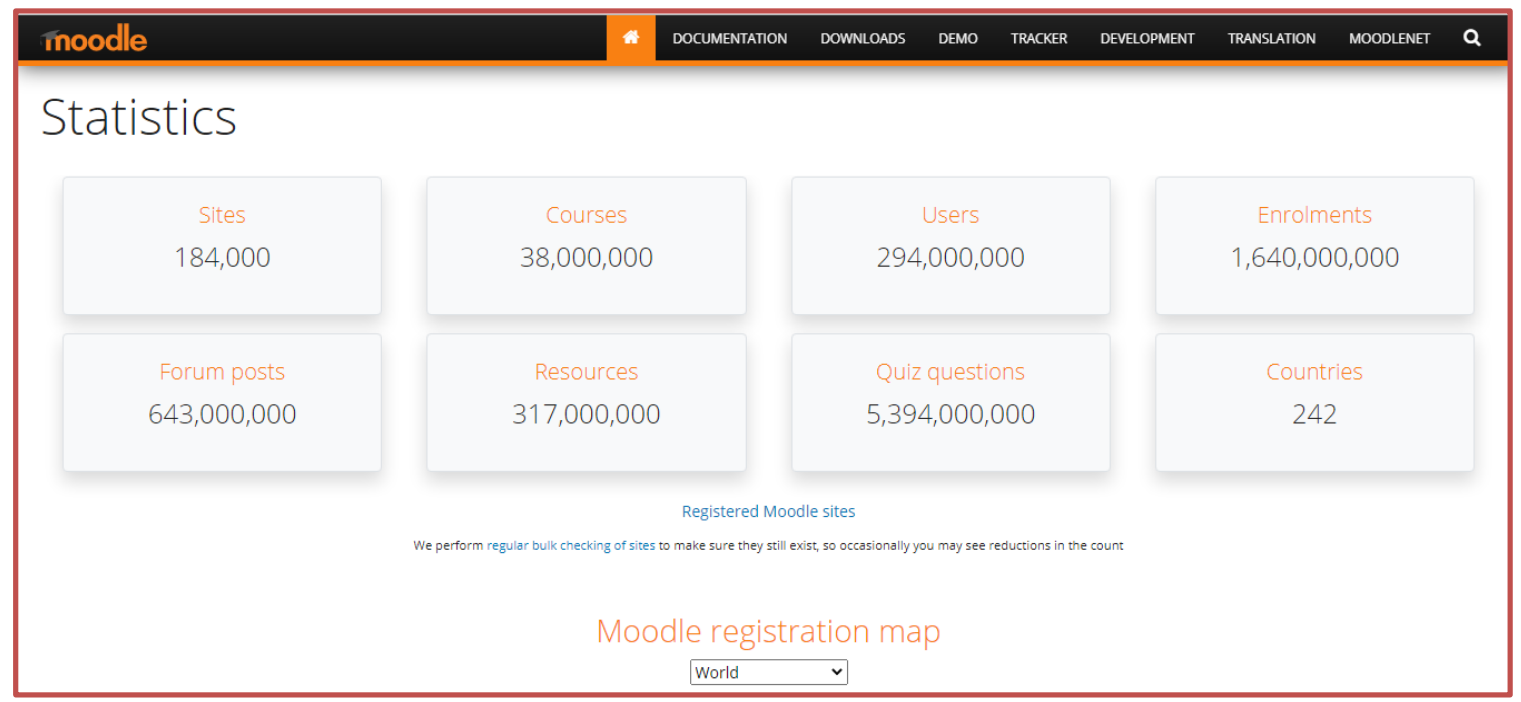

Figura 1. Estadísticas de Moodle en el Mundo.

Nota: Identificación de datos estadísticas globales, presentados en números respecto a los diversos usos de la plataforma Moodle. Adaptada de Moodle, 2021 (https://stats.moodle.org/).

Cada una de las cuales, se analizarán las ventajas competitivas que ofrecen para el aspecto de evaluación. Se señala que, como principal limitante, se utilizará la versión gratuita en el caso de las últimas dos plataformas.

\section{METODOLOGÍA}


Para describir adecuadamente cada una de las herramientas utilizadas para la evaluación significativa y puntual. Se mostrará el uso de las plataformas utilizadas, pero focalizando la atención en los parámetros de evaluación de estas. Indicando además las ventajas del uso de las plataformas. En el presente estudio se utilizaron dichas plataformas en la asignatura de Propiedades de los Materiales en la carrera de Ingeniería Industrial. Es decir, 32 alumnos de segundo semestre, de la carrera de Ingeniería Industrial, con rango de edad de 18 a 19 años.

En primer lugar, se utiliza la plataforma utilizada es Mentimeter, para la evaluación diagnostica. En dicha plataforma se puede crear una cuenta gratuita para realizar consultas online a un grupo de personas y en cuestión de segundos obtener las respuestas en cuestionarios muy fáciles de generar. Por ejemplo, una de las acciones realizadas al inicio del curso es conocer las expectativas de los alumnos. Para ello es necesario diseñar una filmina donde se el cuestionario con opciones de respuesta. Las cuales los alumnos los pueden seleccionar, en la figura 2. Se muestra la utilización de la plataforma para generar una de las preguntas comunes del encuadre de la asignatura.

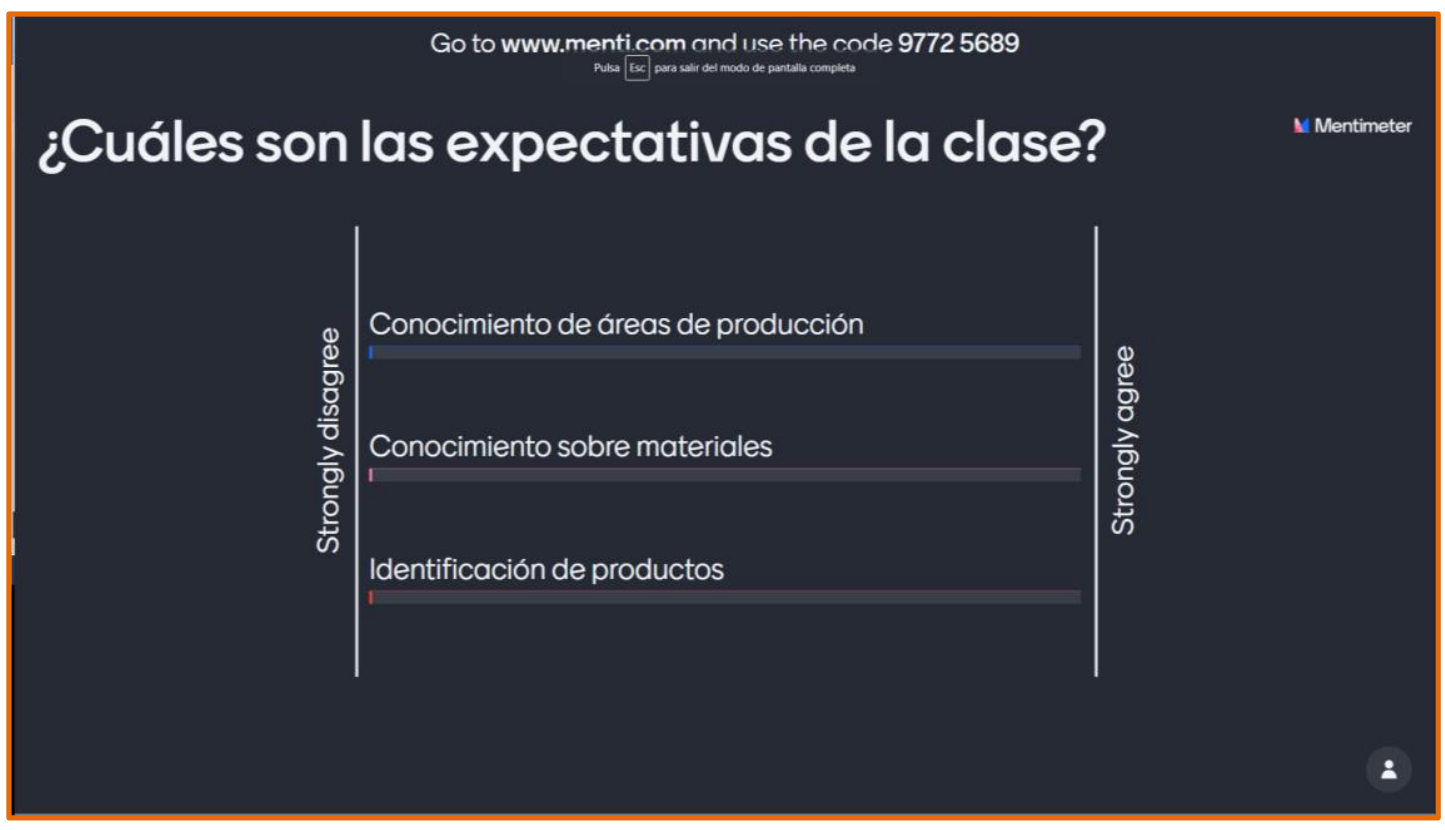

Figura 2. Cuestionario para Encuadre de la Materia Aplicado en Mentimeter.

Nota: Desarrollo de preguntas explorativas, para identificar el nivel de conocimiento generado en el alumno, de acuerdo con sus competencias adquiridas previamente. Adaptada de Mentimeter, 2021 (https://www.mentimeter.com/es-ES).

Para la aplicación del Mentimeter es necesario seguir las instrucciones mencionadas al inicio de la presentación. Se deberá entrar a www.mentimeter.com y colocar el código 97725689. Luego le aparecerá una pantalla como la mostrada en la figura 3. 


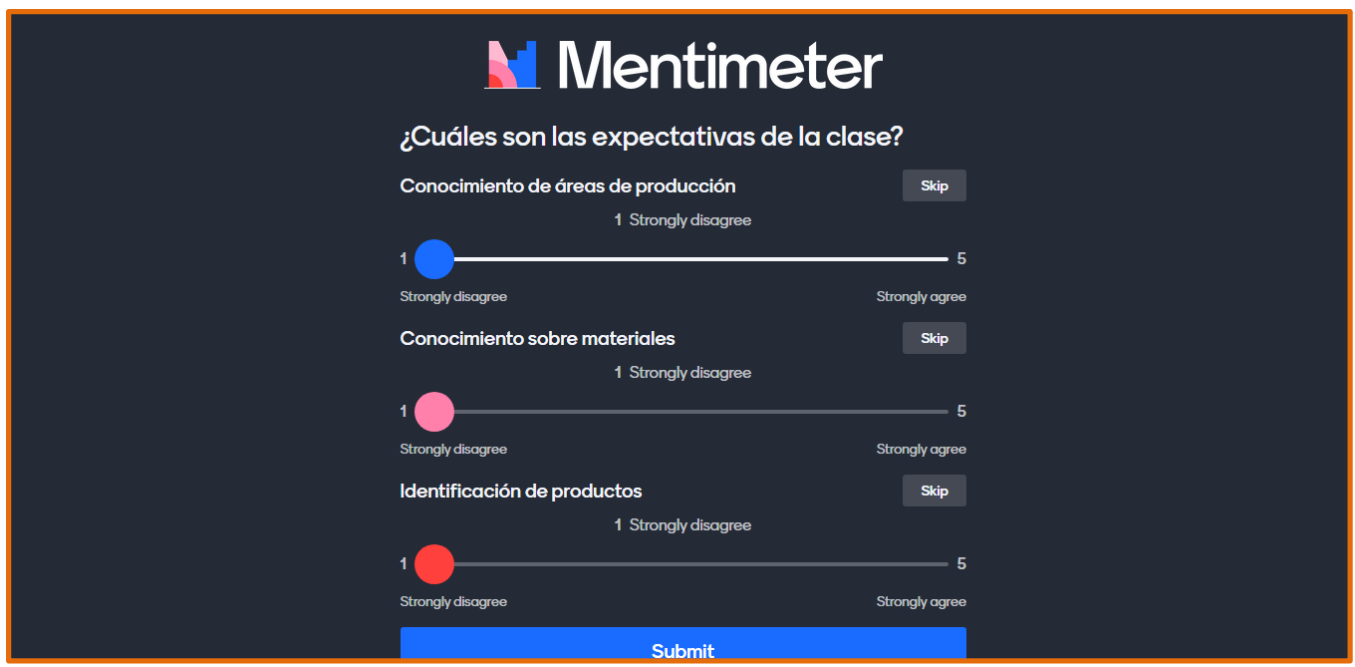

Figura 3. Resultados de la Aplicación de la Evaluación Diagnóstica.

Nota: Representación numérica de los resultados generados en la evaluación diagnóstica e identificación del buen uso de las plataformas digitales por parte del alumno. Adaptada de Mentimeter, 2021

(https://www.mentimeter.com/es-ES).

La evaluación diagnostica resulto esencial para el planteamiento de actividades posteriores e identificar la interacción de los alumnos ante las plataformas digitales. Dando como resultado un impacto positivo, debido a la alta participación de los involucrados.

En segundo lugar, se procedió a realizar la evaluación formativa, para lo cual se utilizó la plataforma Kahoot.com, el periódico El Universal publicó un artículo el día 3 de octubre de 2020, donde indica que esta aplicación, ha resultado ser una herramienta que provee, a docentes y estudiantes, una forma dinámica de aprender y repasar conceptos.

Para su uso se deben seguir los siguientes pasos, en primer punto, se deben registrar como docente y elegir alguna de las versiones, en este caso se eligió la versión gratuita. Enseguida, se debe crear un quiz, para lo que se debe dar un clic en la opción "créate". Luego, conviene completar los campos que la aplicación solicita, tales como título, descripción, tipo de pregunta y límite de tiempo entre otros datos. Dichos campos aparecen en la figura 4, donde se ve la creación de un kahoot común.

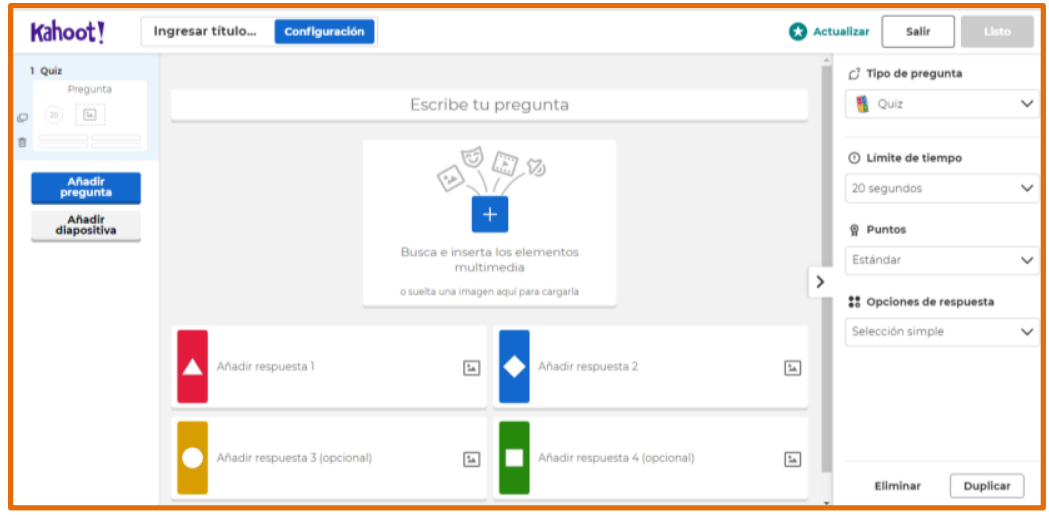

Figura 4. Creación de kahoot para evaluación formativa.

Nota: Representación del desarrollo de las preguntas en la evaluación formativa, aplicadas a los estudiantes mediante la plataforma Kahoot, como parte del proceso de enseñanza. Adaptada de Kahoot, 2021 (https://kahoot.com/). 
Posteriormente se desarrolló un cuestionario de 5 preguntas, para reforzar el conocimiento como parte del proceso de enseñanza. Tras generación del kahoot, se procedió a aplicarlo al grupo antes mencionado, y durante un espacio de 40 minutos se tuvo la dinámica para que ellos fueran contestando una serie de preguntas sobre los elementos analizados en la clase.

Los alumnos en todo momento intentaban participar en el cuestionario puesto que las preguntas aparecían solo durante algunos segundos y ellos tenían que elegir la respuesta correcta. La cual daba una puntuación, ponderando que la respuesta fuera correcta y el tiempo de respuesta a la pregunta. Tras aplicar la prueba a los 20 alumnos arrojó el siguiente resultado, que se muestra en la figura 5.

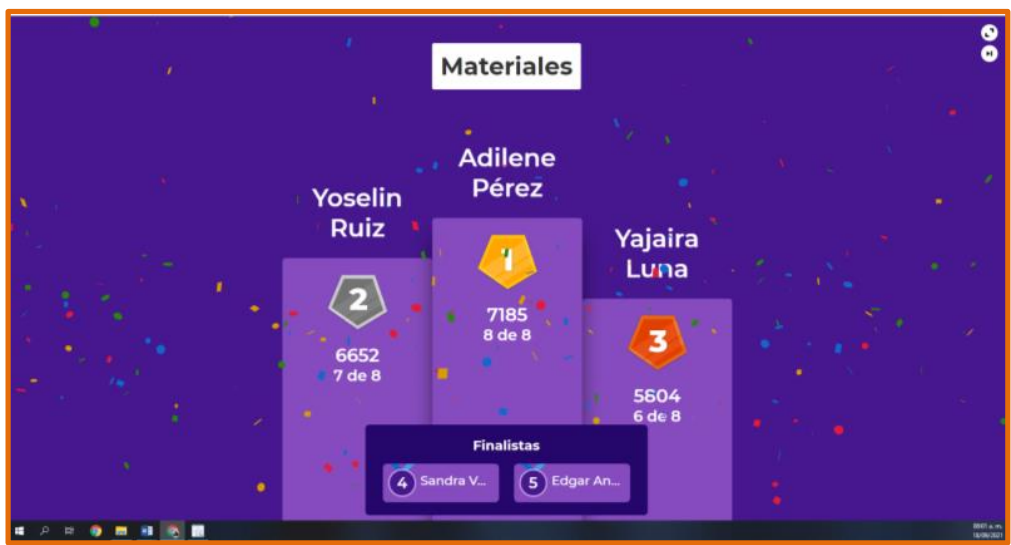

Figura 5. Podium de Respuestas de Evaluación Formativa en Kahoot.

Nota: Representación gráfica de los lugares obtenidos en la evaluación, realizada a los estudiantes mediante la plataforma Kahoot, presentada mediante un podium. Tomado de Kahoot, 2021 (https://kahoot.com/).

Esta prueba dinámica permitió que algunos alumnos aclaran sus dudas previo a la aplicación del examen de la unidad. A los alumnos que quedaron en el pódium, se les otorgaron puntos extra por su desempeño y con el fin de incentivar la participación de las dinámicas propuestas. El uso de esta plataforma como parte de la evaluación formativa generó gran interés de los alumnos e incluso solicitaron fuera aplicada en otras ocasiones.

Y para la parte final, la evaluación sumativa, fue necesario utilizar la última plataforma mencionada, Moodle, la cual es una de las más importantes usadas en la enseñanza a nivel mundial. Cabe resaltar que la asignatura analizada se impartía en el modelo denominado Educación a Distancia, por lo que para los alumnos es esencial el dominio de medios electrónicos. Actualmente el Departamento de Educación a Distancia del ITD cuenta con un dominio para administrar los cursos que se brindan en la especialidad. El dominio es https://plataforma.itdadistancia.tv/ y en ella se encuentra alojado el curso de Propiedades de los materiales.

En dicha plataforma se colocan materiales de consulta, archivos, videos y otros recursos para que el alumno pueda analizarlos y fortalecer su aprendizaje. Sin embargo, aquí es donde el docente tiene la necesidad de realizar evaluaciones para fortalecer el conocimiento de la asignatura. En la figura 6 se muestra la asignatura creada en Moodle. 


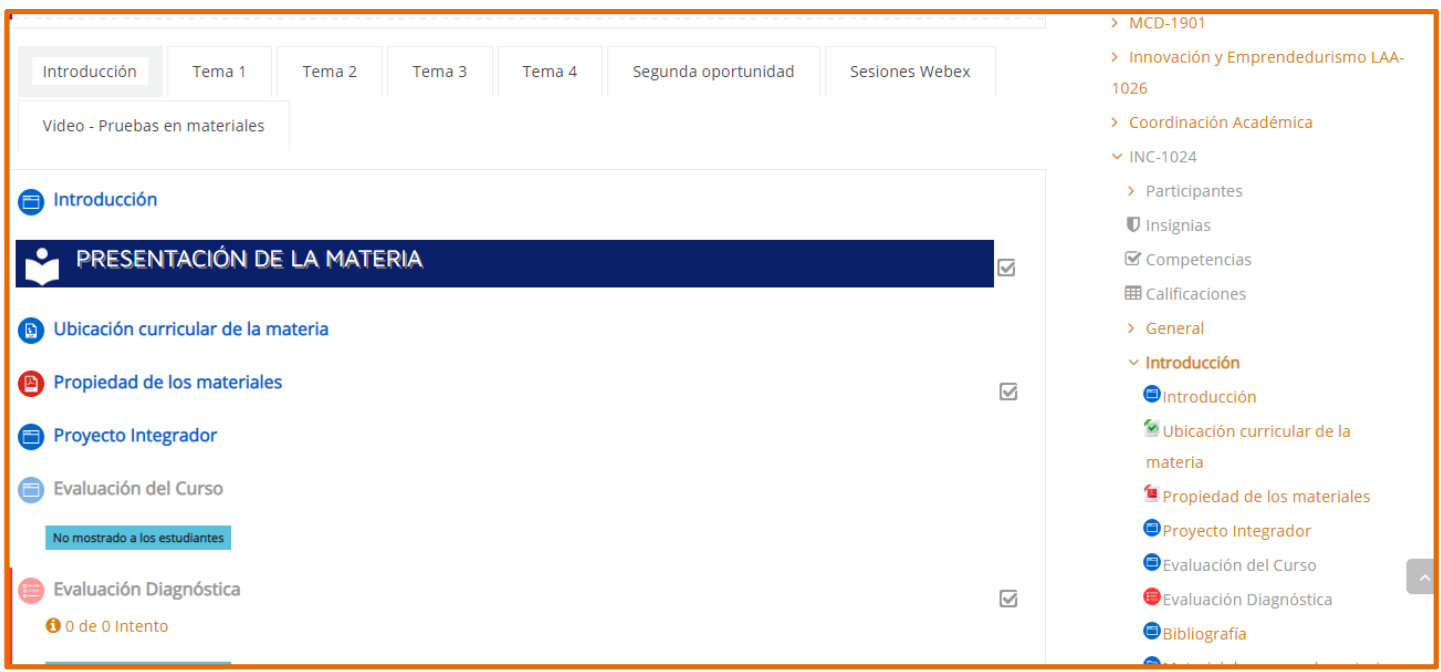

Figura 6. Asignatura de Propiedades de los Materiales en Moodle.

Nota: Desarrollo de materiales de consulta, archivos, videos y otros recursos a través de la plataforma Moodle, generando un fortalecimiento en el aprendizaje del estudiante. Adaptada de Moodle, 2021 (https://plataforma.itdadistancia.tv//).

Entonces llegó el momento de realizar la evaluación en la plataforma Moodle. Para ello fue necesario realizar una actividad denominada Examen. Moodle tiene dos activos muy poderosos denominados actividades y recursos. Es ahí donde se realiza la evaluación, en la plataforma, generando un banco de preguntas, estás se pueden realizar en distintos formatos, como se mencionan a continuación: falso o verdadero, opción múltiple, calculada, etc. En la figura 7 se muestran todas las opciones de preguntas, que contiene el Moodle.

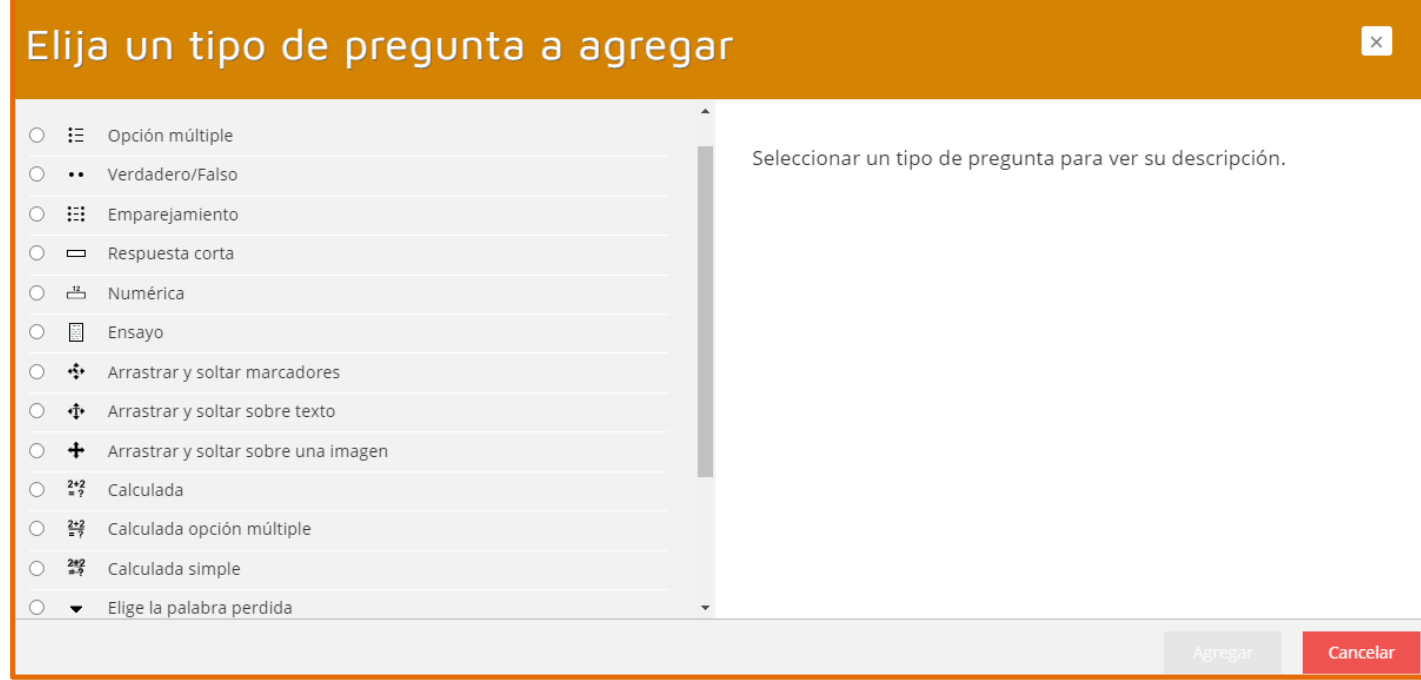

Figura 7. Materia de Propiedades de los Materiales en Moodle.

Nota: Representación de algunos estilos de formatos con los que cuenta la plataforma Moodle, para evaluar los aprendizajes adquiridos. Adaptada de Moodle, 2021 (https://plataforma.itdadistancia.tv//).

Se realizó un banco de preguntas con la teoría analizada en clase, en la figura 8 se muestra parte del banco de preguntas, de manera que se pudiera generar exámenes aleatorios para cada alumno. Se programó para que, incluso las respuestas, aparecieran mezcladas para que de esta manera disminuyeran las probabilidades de que los alumnos se copien las respuestas 
y realmente verificar la incidencia de los métodos usados.

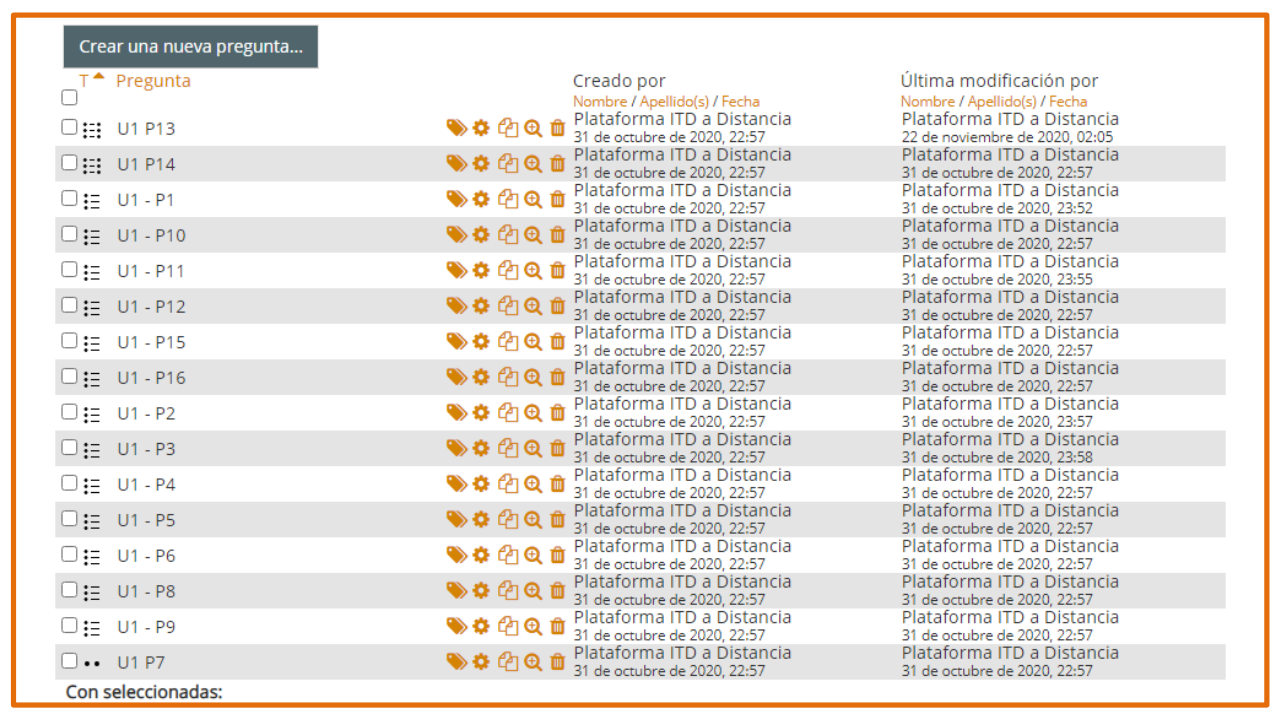

Figura 8. Banco de Preguntas de la Asignatura Propiedades de los Materiales

Nota: Identificación del desarrollo del banco de preguntas generando exámenes aleatorios para cada alumno e incluso las respuestas, de esta manera disminuir las probabilidades para que los alumnos se copien las respuestas y realmente verificar la incidencia de los métodos usados. Adaptada de Moodle, 2021

(https://plataforma.itdadistancia.tv//).

Finalmente se obtuvieron las calificaciones de los alumnos para el tema 1, y se observa que la gran mayoría de los alumnos acreditaron el examen. En la figura 9 se muestra el resultado arrojado por la plataforma.

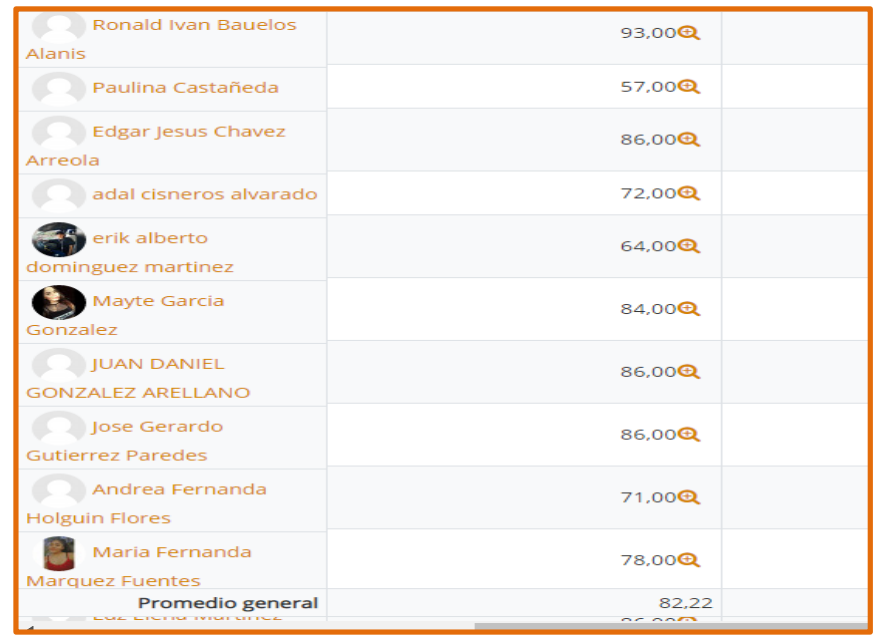

Figura 9. Materia de Propiedades de los Materiales en Moodle

Nota: Muestra de calificaciones de los alumnos para el tema 1, la gran mayoría de los alumnos acreditaron el examen. Adaptada de Moodle, 2021 (https://plataforma.itdadistancia.tv//).

Ahora solo queda hacer el contraste con las anteriores ocasiones en las cuales se había impartido la asignatura y contrastar los resultados. 


\section{RESULTADOS}

Considerando la importancia de esta investigación, de acuerdo con Gómez (2004), afirma que las tecnologías constituyen un medio como jamás ha existido, ofreciendo un acceso instantáneo a la información. También afirma que a cada individuo le toca enriquecer y construir su saber a partir de esa información y a la educación le corresponde proporcionar las bases para que esto se produzca.

Por lo que, se puede apreciar en los resultados que las herramientas digitales, como lo son las plataformas en estudio Moodle, Mentimeter y Kahoot, ayudan a mejorar el aprendizaje de aspectos básicos de la asignatura.

Haciendo una comparativa entre el método convencional y el uso actual de herramientas digitales para el fortalecimiento del proceso de enseñanza, además de la evaluación. Desde el año 2012 se habían presentado altos índices de reprobación, los índices de reprobación fluctuaban en la primera unidad desde el 30\% hasta el $60 \%$, sin embargo, a partir del 2020 que se utilizaron estas y algunas otras plataformas, el índice de reprobación bajo a $10 \%$ en el primer grupo y en el caso del grupo 2 se obtuvo un $9 \%$.

Durante la última vez que se impartió la asignatura estos fueron los resultados que se presentaron, tal y como se muestra en la figura 10.

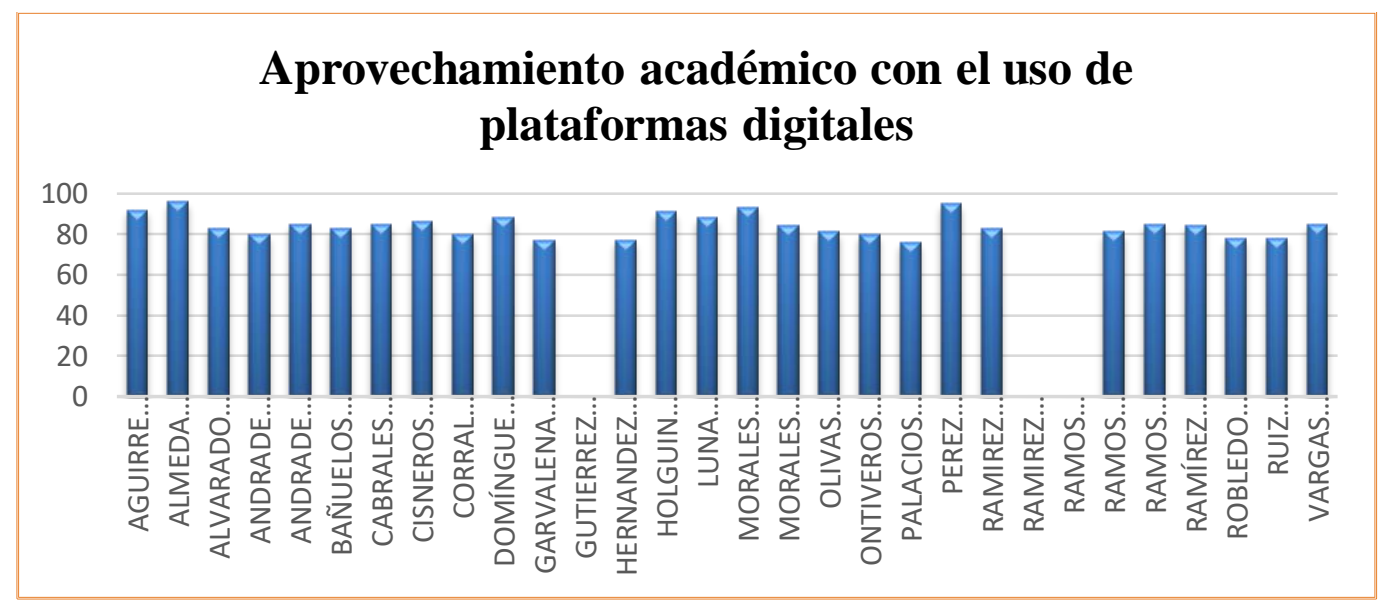

Figura 10. Resultados de las Evaluaciones de la Asignatura Propiedades de los Materiales en Moodle

Nota: Representación gráfica de los resultados grupales de la última vez que se impartió la asignatura "Propiedades de los Materiales en Moodle. Adaptada de Moodle, 2021 (https://plataforma.itdadistancia.tv//).

Los resultados anteriores permiten ver el impacto positivo en la evolución de los alumnos de la clase de Propiedades de los Materiales, impartida en el Instituto Tecnológico de Durango para la carrera de Ingeniería Industrial en su modalidad de Educación a Distancia.

\section{CONCLUSIONES}

En base a la investigación realizada, es razonable concluir que se pueden utilizar e incorporar las plataformas digitales educativas para los tres momentos de la evaluación; la evaluación diagnostica, la evaluación formativa y la evaluación sumativa. Es fácil su incorporación al proceso de enseñanza-aprendizaje, debido a que resultan atractivas e interesantes para el 
aprendizaje de los alumnos. Cuando el alumno repasa temas de asignaturas tan complejas con métodos distintos a los tradicionales, logra una mejor adopción de los conceptos, lógicamente esto genera una mejora en el índice de aprobación, particularmente abatiendo el índice en más de un $20 \%$ con respecto a los últimos años.

Esto permite inferir que, se puede seguir innovando en la forma de evaluar, los tres momentos de evaluación del proceso de enseñanza-aprendizaje por medio de medios digitales, y no pensar que estos deben seguir métodos comunes y trillados. Por lo que se logró mejorar el índice de aprobación, al usar plataformas digitales en el proceso enseñanza aprendizaje.

Es necesario en esta época de la digitalización, que se busquen nuevas herramientas de evaluación que permitan una mayor interacción con los alumnos, debido a que, al realizarse las asesorías virtuales, es muy fácil perder la atención de los alumnos, ocasionado por que algunos docentes, siguen impartiendo cátedras tradicionales, también se ve obstaculizada la relación tradicional incluida en el modelo de educación a Distancia. Por lo que se deberían utilizar diversas herramientas en la enseñanza universitaria, pero sobre todo en la evaluación de los aprendizajes, para proponer soluciones a las problemáticas presentadas y generar un conocimiento significativo en la educación a distancia.

Para hacer uso de las tecnologías propuestas el docente debe mostrar un interés en capacitarse en áreas distintas a las que él conoce y domina, evitar con ello la resistencia al cambio, que le ayudarán a incrementar el aprendizaje y por consiguiente que exista un mejor índice de aprobación.

Claro está que, la mayor retribución que tiene el docente que se actualiza es el impacto final que tienen sus métodos y técnicas en el aprendizaje de los alumnos, por lo que, será necesario contrastar los resultados iniciales con los posteriores, para analizar las áreas de oportunidad que se presenten.

\section{BIBLIOGRAFÍA}

ACUÑA, S.R. Y WEBER, V. (1999): "La utilización de hipertextos como herramientas para el aprendizaje y la instrucción", en Las Nuevas Tecnologías para la Mejora Educativa. Edutec'99. Kronos. Sevilla. Pp. 175-188.

ADELL (1997): "Tendencias en educación en la sociedad de las tecnologías de la información", Revista EDUTEC. Noviembre 1997, nº 7.

ADELL, J. (1998b): "Redes y educación, en DE PABLOS, J. y JIMÉNEZ, J. (Eds.): Nuevas tecnologías, comunicación audiovisual y educación. Ed. Cadecs. Barcelona. pp: 177-211.

ADELL, J. y GISBERT, M. (1998a): "Educació a Internet: l'aula virtual". Temps d'Educació. Universitat de Barcelona. No 18. pp: 263-277.

ALONSO, C.M; GALlEGO, D.J.; HONEY, P. (1999): Los estilos de aprendizaje. Procedimientos de Diagnóstico y Mejora. Ediciones Mensajero, Bilbao. $4^{\mathrm{a}}$ Edición

AREA, M. (2003): Creación y uso de webs para la docencia universitaria. Departamento de Didáctica e Investigación Educativa y del Comportamiento. Facultad de Educación. Universidad de la Laguna.

http://webpages.ull.es/users/manarea/biblioteca.htm [consultado 1 de septiembre de 2003] 
BARAJAS, M., SCHEUERMANN, F. Y KIKIS, K (2003): “¿Peligra el papel del profesor como autoridad del conocimiento en un entorno de aprendizaje a través de las TIC?", en Elearning Europa: http://www.elearningeuropa.info [consultado 02/05/2003]

BARBERÁ. E. (Coord.) (2001): La incógnita de la educación a distancia. Ice de la Universidad de Barcelona-Editorial Horsori. Cuadernos de educación. Barcelona.

BARCELÓ, A. y otros (2000): "El apoyo técnico y pedagógico en la utilización de las Tecnólogas de la Información y Comunicación en el contexto universitario", en III Congreso Internacional de Comunicación, Tecnología y Educación-Redes, multimedia y diseños virtuales. Oviedo (sin paginar). 